\title{
LOCAL TIME DECAY FOR A NONLINEAR BEAM EQUATION *
}

\author{
J. E. $\operatorname{LIN}^{\dagger}$
}

Abstract. Using the Morawetz' Radial Identity, we show that the local energy of a solution is integrable in time and the local $L^{2}$ norm of the solution approaches zero as time approaches the infinity for a nonlinear beam equation with the spatial dimension $>5$.

1. Introduction. Consider a nonlinear beam equation

$$
u_{t t}+\Delta^{2} u+f(u)=0
$$

where $u=u(x, t), x=\left(x_{1}, x_{2}, \ldots, x_{n}\right) \in R^{n}, R^{n}$ is the n-dimensional Euclidean space, $n>5, t \geq 0, \Delta=$ Laplacian in $x$, and $f(u)$ satisfies

$$
c_{1}(u f(u)-2 F(u))+c_{0} u^{2} \geq F(u) \geq c_{0} u^{2}
$$

for some positive constants $c_{0}$ and $c_{1}$, where $F^{\prime}(u)=f(u)$ with $F(0)=0$. As usual, the subscript in variables denotes the partial derivative, thus, $u_{t}=\partial u / \partial t$, etc... We also use the notations $\partial_{j}=\partial / \partial x_{j}$, and $u_{r}=(x / r) \cdot \nabla u$, where $\nabla$ is the gradient in $x$, and $r=|x|$. Moreover, for a function of one variable $g(s), g^{\prime}(s)=d(g(s)) / d s$ denotes the derivative of $g$ in $s$. Finally, that a function is $C^{n}$ means that its $n^{\text {th }}$ partial derivatives are continuous. In this work, we show that the local energy of a solution is integrable in time and the local $L^{2}$ norm of a solution approaches zero as time approaches the infinity. Our method follows [1] in utilizing the Morawetz' Radial Identity [5].

The global scattering problem was considered in [1] along with several inequalities. It was conjectured in [1] that the local energy is integrable in $t$ and tends to zero as $t$ approaches the infinity. This work proves the first part of this conjecture. The well-posedness, low-energy scattering, stability and instability of solitary and standing waves, and the time decay of solutions for the nonlinear beam equation with a slightly different $f(u)$ can be found in $[2,3]$. In the one-spatial dimension, a similar equation to (1) with a different nonlinear term has been studied as a model for a suspension bridge [4].

We shall need the following result from [1]:

The energy $E[u]=\int_{R^{n}}\left[(1 / 2) u_{t}^{2}+(1 / 2)|\Delta u|^{2}+F(u)\right] d x$ is a constant, and for $n>5$, assuming that $u$ is a solution that is smooth enough and small enough at the spatial infinity, then there is a positive constant $c$ such that

$$
\begin{gathered}
\int_{0}^{\infty} \int_{R^{n}}(1 / r)[u f(u)-2 F(u)] d x d t \leq c E[u] \\
\int_{0}^{\infty} \int_{R^{n}}\left(1 / r^{3}\right)|\nabla u|^{2} d x d t \leq c E[u], \text { and } \\
\int_{0}^{\infty} \int_{R^{n}}\left(1 / r^{5}\right)|u|^{2} d x d t \leq c E[u], \text { provided } n \geq 6
\end{gathered}
$$

\footnotetext{
* Received January 7, 2004; accepted for publication May 5, 2004.

$\dagger$ Department of Mathematical Sciences, George Mason University, Fairfax, VA 22030, USA (jelin@gmu.edu).
} 
2. The Morawetz' radial identity. Let $\zeta$ be a $C^{4}$ radially symmetric function of $x$. Multiplying $(1)$ by $\zeta\left(u_{r}+((n-1) /(2 r)) u\right)$ we get the following identity, assuming that $u$ is $C^{2}$ in $t$ and $C^{4}$ in $x$,

$$
\begin{aligned}
0 & =\left(u_{t t}+\Delta^{2} u+f(u)\right) \zeta\left(u_{r}+(n-1) u /(2 r)\right) \\
& =X_{t}+\nabla \cdot Y+Z
\end{aligned}
$$

where

$X=u_{t} \zeta\left(u_{r}+(n-1) u /(2 r)\right)$,

$Y=-\left((n-1) u^{2} / 4\right)(\nabla(\Delta(\zeta / r)))+$ a function depending on $\zeta, \zeta^{\prime}, \zeta^{\prime \prime}, \zeta^{\prime \prime \prime}, x, u, F(u)$, $\nabla u, \Delta u, \nabla(\Delta u), u_{r}, \nabla u_{r}, u_{t}, 1 / r, 1 / r^{2}$ and $1 / r^{3}$,

and

$$
\begin{aligned}
Z= & \left(\zeta^{\prime} / 2\right)\left(u_{t}\right)^{2}+(\Delta u)^{2}\left[3 \zeta^{\prime} / 2\right]+A\left(u_{r}\right)^{2}+B\left(|\nabla u|^{2}-\left(u_{r}\right)^{2}\right)+C u^{2}+(n-1) \zeta(u f(u) \\
& -2 F(u)) /(2 r)-\zeta^{\prime} F(u)+\left(\zeta-r \zeta^{\prime}\right) P,
\end{aligned}
$$

where

$$
\begin{aligned}
& A=-7 \zeta^{\prime \prime \prime} / 2-\zeta^{\prime}(n-1)(n-3) /\left(2 r^{2}\right)+\zeta(n-1)(n-3) /\left(2 r^{3}\right), \\
& B=-3 \zeta^{\prime \prime \prime} / 2+\zeta^{\prime \prime}(n-5) / r-\zeta^{\prime}\left(n^{2}+2 n-19\right) /\left(2 r^{2}\right)+\zeta\left(n^{2}+2 n-19\right) /\left(2 r^{3}\right), \\
& C=((n-1) / 2)\left[\zeta^{\prime \prime \prime \prime \prime \prime} /(2 r)+\zeta^{\prime \prime \prime}(n-3) /\left(r^{2}\right)+\zeta^{\prime \prime}(n-3)(n-7) /\left(2 r^{3}\right)-3 \zeta^{\prime}(n-3)(n\right. \\
&\left.-5) /\left(2 r^{4}\right)+3 \zeta(n-3)(n-5) /\left(2 r^{5}\right)\right], \\
& P=(2 / r)\left[\sum_{i, j}\left(S_{i j} u\right)^{2}-\sum_{i}\left(\sum_{j}\left(x_{j} / r\right) S_{i j} u\right)^{2}\right] \geq 0 \\
& \text { with } S_{i j} u=\left(\left(x_{i}\right) / r^{3}\right) \sum_{k}\left[x_{k}\left(x_{k} \partial_{j}-x_{j} \partial_{k}\right) u_{r}\right]+\partial_{j} \sum_{k}\left[\left(x_{k} / r^{2}\right)\left(x_{k} \partial_{i}-x_{i} \partial_{k}\right) u\right] .
\end{aligned}
$$
of $[1]$.

REMARK. If $\zeta=1$, this identity is the identity shown in the proof of Theorem 1

3. The integrability of the local energy. We now state the main result of this work.

Theorem. Consider the nonlinear beam equation (1) with the condition (2) on $f(u)$. Assume that the spatial dimension $n$ is $>5$. Assume also that $u$ is $C^{2}$ in $t$ and $C^{4}$ in $x, u_{t}$ is bounded, and $u$ and all its partial derivatives in $x$ up to the $4^{\text {th }}$ order approach zero as $|x|$ approaches the infinity. Then the local energy is integrable in $t$.

Proof. Assume that $\zeta$ and $\zeta^{\prime}$ are non-negative functions and $\zeta, \zeta^{\prime}, \zeta^{\prime \prime}$, and $\zeta^{\prime \prime \prime}$ are bounded functions. We integrate both sides of (6) with respect to $x$ in $R^{n}$ and $t$ from 0 to $T$. With the assumption on the smoothness and smallness of $u$ at the spatial infinity, we have $\int_{R^{n}} \nabla \cdot Y d x=0$ for $n>5$.

Thus

$$
0 \geq \int_{R^{n}} X(x, T) d x-\int_{R^{n}} X(x, 0) d x+\int_{0}^{T} \int_{R^{n}} Z d x d t .
$$

Now we can rewrite $X$ into two ways:

$$
\begin{aligned}
X= & -(\zeta / 2)\left(u_{t}^{2}+|\nabla u|^{2}\right)+(\zeta / 2)\left[u_{t}^{2}+|W|^{2}+2((x / r) \cdot W) u_{t}\right] \\
& -\nabla \cdot\left[(n-1) \zeta u^{2} x /\left(4 r^{2}\right)\right]+(n-1)(n-3) \zeta u^{2} /\left(8 r^{2}\right)+(n-1) \zeta^{\prime} u^{2} /(4 r),
\end{aligned}
$$


and

$$
\begin{aligned}
X= & (\zeta / 2)\left(u_{t}^{2}+|\nabla u|^{2}\right)-(\zeta / 2)\left[u_{t}^{2}+|W|^{2}-2((x / r) \cdot W) u_{t}\right] \\
& +\nabla \cdot\left[(n-1) \zeta u^{2} x /\left(4 r^{2}\right)\right]-(n-1)(n-3) \zeta u^{2} /\left(8 r^{2}\right)-(n-1) \zeta^{\prime} u^{2} /(4 r),
\end{aligned}
$$

where $\mathrm{W}=\nabla u+(n-1) u x /\left(2 r^{2}\right)$.

Using the first way ( 7$)$ for $X(x, T)$ and the second way (8) for $X(x, 0)$, we get

$$
\int_{R^{n}}(1 / 2) \zeta\left(u_{t}^{2}+|\nabla u|^{2}\right)(x, 0) d x+\int_{R^{n}}(1 / 2) \zeta\left(u_{t}^{2}+|\nabla u|^{2}\right)(x, T) d x \geq \int_{0}^{T} \int_{R^{n}} Z d x d t .
$$

Let $T$ approach the infinity, we get

$$
\int_{0}^{\infty} \int_{R^{n}} Z d x d t \leq c_{2} E[u], \text { for some positive constant } c_{2},
$$

where $c_{2}$ depends on $c_{0}, c_{1}$ and the bound for $\zeta$.

Now, let $\zeta(x)=\zeta(r)=1-1 /\left(r^{2}+4\right)^{2}$, where $r=|x|$. Since $\zeta$ and $\zeta^{\prime}$ are non-negative and $\zeta, \zeta^{\prime}, \zeta^{\prime \prime}$, and $\zeta^{\prime \prime \prime}$ are bounded functions, the inequality (9) holds. Substituting $\zeta$ into $(9)$, we get

$$
\begin{aligned}
& \int_{0}^{\infty} \int_{R^{n}}\left[\left(u_{t}^{2}+(\Delta u)^{2}\right) r /\left(r^{2}+4\right)^{3}+|\nabla u|^{2} /\left(r^{3}\left(r^{2}+4\right)^{5}\right)+P /\left(r^{2}+4\right)^{3}\right. \\
& \left.+u^{2} /\left(r^{5}\left(r^{2}+4\right)^{6}\right)+(u f(u)-2 F(u)) /\left(r\left(r^{2}+4\right)^{2}\right)\right] d x d t \\
\leq & \int_{0}^{\infty} \int_{R^{n}} 4 F(u) r /\left(r^{2}+4\right)^{3} d x d t+c_{2} E[u] \\
\leq & \int_{0}^{\infty} \int_{R^{n}}\left[4 c_{1}(u f(u)-2 F(u))+4 c_{0} u^{2}\right] r /\left(r^{2}+4\right)^{3} d x d t+c_{2} E[u] \\
\leq & \int_{0}^{\infty} \int_{R^{n}}\left[4 c_{1}(u f(u)-2 F(u)) / r+4 c_{0} u^{2} / r^{5}\right] d x d t+c_{2} E[u] \\
\leq & c_{3} E[u]
\end{aligned}
$$

for some positive constant $c_{3}$. Note that we have used (3) and (5) in the above inequality.

Let $h>b>0$, we get

$$
\int_{0}^{\infty} \int_{b \leq|x| \leq h}\left[u_{t}^{2}+(\Delta u)^{2}+|\nabla u|^{2}+u^{2}+(u f(u)-2 F(u))\right] d x d t \leq c_{4} E[u]
$$

for some positive contant $c_{4}$ depending on $b$ and $h$.

Therefore, $\int_{0}^{\infty} \int_{b \leq|x| \leq h}\left[u_{t}^{2}+(\Delta u)^{2}+F(u)\right] d x d t \leq c_{5} E[u]$, for some positive constant $c_{5}$ depending on $c_{0}, c_{1}, b$ and $h$. Since the equation (1) is invariant under spatial translation, we get

$$
\int_{0}^{\infty} \int_{|x| \leq h}\left[u_{t}^{2}+(\Delta u)^{2}+F(u)\right] d x d t \leq c_{6} E[u] \text {, for some positive constant } c_{6} \text {. }
$$

Therefore the local energy is integrable in time. 
4. Time decay of the local $L^{2}$ norm. Now, we are going to show that the local $L^{2}$ norm of $u$ approaches 0 as $t$ approaches the infinity. The idea of the proof is from [5]. Let $h>0$ and $t>t_{1}>0$, then

$$
\begin{aligned}
\left(t-t_{1}\right) \int_{|x| \leq h} u^{2}(x, t) d x & =\int_{t_{1}}^{t} \partial\left[\left(\tau-t_{1}\right) \int_{|x| \leq h} u^{2}(x, \tau) d x\right] / \partial \tau d \tau \\
& =\int_{t_{1}}^{t} \int_{|x| \leq h} u^{2}(x, \tau) d x d \tau+\int_{t_{1}}^{t}\left(\tau-t_{1}\right) \int_{|x| \leq h} 2 u u_{t}(x, \tau) d x d \tau .
\end{aligned}
$$

Let $t_{1}=t-1$, we get

$$
\int_{|x| \leq h} u^{2}(x, t) d x \leq \int_{t-1}^{t} \int_{|x| \leq h} u^{2}(x, \tau) d x d \tau+\int_{t-1}^{t} \int_{|x| \leq h}\left(u^{2}+u_{t}^{2}\right)(x, \tau) d x d \tau .
$$

Hence $\int_{|x| \leq h} u^{2}(x, t) d x$ approaches 0 as $t$ approaches the infinity since the local energy is integrable in time.

Acknowledgement. The author wishes to thank Professor W. A. Strauss for the stimulating discussion about this equation.

\section{REFERENCES}

[1] S. P. Levandosky and W. A. Strauss, Time Decay for the Nonlinear Beam Equation, Methods and Applications of Analysis, 7 (2000), pp. 479-488.

[2] S. P. Levandosky, Stability and Instability of Fourth-order Solitary Waves, J. Dynam. Diff. Equations., 10 (1998), pp. 151-188.

[3] S. P. Levandosky, Decay Estimates for Fourth-order Wave Equations, J. Differential Equations, 143 (1998), pp. 360-413.

[4] P. J. Mckenna And W. Walter, Traveling Waves in a Suspension Bridge, SIAM J. Appl. Math., 50 (1990), pp. 703-715.

[5] C. S. Morawetz, Time Decay for the Nonlinear Klein-Gordon Equation, Proc. Roy. Soc. A, 306 (1968), pp. 291-296. 